\title{
Co-expression network analysis reveals the pivotal role of mitochondrial dysfunction and interferon signature in juvenile dermatomyositis
}

\author{
Danli Zhong ${ }^{1,2}$, Chanyuan Wu ${ }^{1,2}$, Jingjing Bai ${ }^{1,2}$, Dong Xu ${ }^{1,2}$, Xiaofeng Zeng ${ }^{1,2}$, Qian Wang ${ }^{\text {Corresp. } 1,2}$ \\ ${ }^{1}$ Department of Rheumatology, Peking Union Medical College Hospital, Peking Union Medical College \& Chinese Academy of Medical Sciences; National \\ Clinical Research Center for Dermatologic and Immunologic Diseases (NCRC-DID), Beijing, China \\ 2 Key Laboratory of Rheumatology and Clinical Immunology, Ministry of Education, Beijing, China \\ Corresponding Author: Qian Wang \\ Email address: wangqian@pumch.cn
}

Background: Juvenile dermatomyositis (JDM) is an immune-mediated disease characterized by chronic organ inflammation. The pathogenic mechanisms remain illdefined; Methods: Raw microarray data of JDM were obtained from the gene expression omnibus (GEO) database. Based on GSE3307 dataset with 39 samples, weighted correlation network analysis (WGCNA) was performed to identify key modules associated with pathological state. Functional enrichment analyses were conducted to identify potential mechanisms. Based on the criteria of high connectivity and module membership, candidate hub genes were selected. A protein-protein interaction network was constructed to identify hub genes. Another dataset (GSE11971) was used for the validation of real hub genes. Finally, the real hub genes were used to screen out small-molecule compounds via the Connectivity map database ; Results: Three modules were considered as key modules for the pathological state of JDM. Functional enrichment analysis indicated that responses to interferon and metabolism were dysregulated. A total of 45 candidate hub genes were selected according to the pre-established criteria, and 20 genes could differentiate JDM from normal controls by validation of another external dataset (GSE11971). These real hub genes suggested the pivotal role of mitochondrial dysfunction and interferon signature in JDM. Furthermore, drug repositioning highlighted the importance of acacetin, helveticoside, lanatoside C, deferoxamine, LY-294002, tanespimycin and L01AD from downregulated genes with the potential to perturb the development of JDM, while betonicine, felodipine, valproic acid, trichostatin $A$ and sirolimus from upregulated genes provided potentially therapeutic goals for JDM; Conclusions: There are 20 real hub genes associated with the pathological state of JDM, suggesting the pivotal role of mitochondrial dysfunction and interferon signature in JDM. This analysis predicted several kinds of smallmolecule compounds to treat JDM. 
1 Co-expression network analysis reveals the pivotal role of 2 mitochondrial dysfunction and interferon signature in 3 juvenile dermatomyositis

4

5

6

Danli Zhong ${ }^{1}$, Chanyuan $\mathrm{Wu}^{1}$, Jingjing $\mathrm{Bai}^{1}$, Dong $\mathrm{Xu}^{1}$, Xiaofeng Zeng ${ }^{1}$, Qian Wang ${ }^{1, *}$

${ }^{1}$ Department of Rheumatology, Peking Union Medical College Hospital, Peking Union Medical College \& Chinese Academy of Medical Sciences; National Clinical Research Center for Dermatologic and Immunologic Diseases (NCRC-DID); Key Laboratory of Rheumatology and Clinical Immunology, Ministry of Education, Beijing, China.

Corresponding Author:

Qian Wang ${ }^{1, *}$

Department of Rheumatology, Peking Union Medical College Hospital, Peking Union Medical College \& Chinese Academy of Medical Sciences; National Clinical Research Center for Dermatologic and Immunologic Diseases (NCRC-DID); Key Laboratory of Rheumatology and Clinical Immunology, Ministry of Education. No.1 Shuaifuyuan, Beijing 100730, China.

Email address: wangqian@pumch.cn

\section{Abstract}

Background: Juvenile dermatomyositis (JDM) is an immune-mediated disease characterized by chronic organ inflammation. The pathogenic mechanisms remain ill-defined; Methods: Raw microarray data of JDM were obtained from the gene expression omnibus (GEO) database. Based on GSE3307 dataset with 39 samples, weighted correlation network analysis (WGCNA) was performed to identify key modules associated with pathological state. Functional enrichment analyses were conducted to identify potential mechanisms. Based on the criteria of high connectivity and module membership, candidate hub genes were selected. A protein-protein interaction network was constructed to identify hub genes. Another dataset (GSE11971) was used for the validation of real hub genes. Finally, the real hub genes were used to screen out smallmolecule compounds via the Connectivity map database; Results: Three modules were considered as key modules for the pathological state of JDM. Functional enrichment analysis indicated that responses to interferon and metabolism were dysregulated. A total of 45 candidate hub genes were selected according to the pre-established criteria, and 20 genes could differentiate JDM from normal controls by validation of another external dataset (GSE11971). These real hub genes suggested the pivotal role of mitochondrial dysfunction and interferon signature in JDM. Furthermore, drug repositioning highlighted the importance of acacetin, helveticoside, lanatoside C, deferoxamine, LY-294002, tanespimycin and L01AD from downregulated genes with the potential to perturb the development of JDM, while betonicine, felodipine, valproic acid, trichostatin A and sirolimus from upregulated genes provided potentially therapeutic goals for JDM; Conclusions: There are 20 real hub genes associated with the pathological state of JDM, 
40 suggesting the pivotal role of mitochondrial dysfunction and interferon signature in JDM. This 41 analysis predicted several kinds of small-molecule compounds to treat JDM.

42 Keywords: juvenile dermatomyositis; weighted gene co-expression network analysis; hub genes; 43 small-molecule compounds

\section{Introduction}

Juvenile dermatomyositis (JDM) is a rare chronic childhood-onset autoimmune disease characterized by inflammatory infiltration in small vessels and tissues within skin and muscle. The incidence of JDM is 2-4 per million per year in the United States (Feldman et al. 2008), with female:male ratios ranging from 1.5:1 to 5:1 (Lindsley et al. 1995). The major manifestations of JDM patients consist of symmetrical proximal muscle weakness, skin rashes, and internal organs involvement (Crowe et al. 1982). Up to $30 \%$ of JDM may present with calcifications, one of the prognostic factors of long-term disability (Arabshahi et al. 2012; Li \& Zhou 2019; Ravelli et al. 2010). Adults with JDM in childhood are susceptible to premature cardiovascular damage (Gitiaux et al. 2016).

Pathological state and treatment have been reported to affect growth and puberty in the active phase of JDM (Nordal et al. 2019). Ongoing disease activity, irreversible damage, and aggressive immunosuppressive therapy remain major challenges for long-term outcomes and quality of life in JDM patients (Hoeltzel et al. 2014). The etiology of JDM remains ill-defined although genetic and environmental factors are suspected to be involved in its pathogenesis. It has been reported that JDM patients had higher incidence of Epstein-Barr virus infection (Zheng et al. 2019), and the prominent type 1 interferon (IFN) signature was shown to affect the vasculature JDM (De Paepe 2017; Greenberg 2010). Adaptive and innate immune mechanisms involving IFNassociated molecules appear to mediate endothelial tubule-reticular formations and peri-fascicular atrophy.

Weighted gene co-expression network analysis (WGCNA) algorithm is a powerful bioinformatic method that mines practical information from gene expression profiles by constructing of gene modules, thereby interpreting the biological significance of a gene (Langfelder \& Horvath 2008). WGCNA has been widely used in various diseases (Zhao et al. 2010), including malignancies, cardiovascular diseases and autoimmune diseases, where it has provided useful information for understanding pathological process and for discovery of diagnostic and prognostic biomarkers. Nevertheless, WGCNA has never been applied to JDM.

Therefore, we used WGCNA for the first time to analyze pathological state and gene expression data in JDM muscular samples to explore and validate hub genes associated with JDM, as well as to predict small-molecule compounds to treat JDM with promising perspectives.

\section{Materials \& Methods}

\section{Data Collection and Differentially Expressed Genes Screening}

The flowchart of the study is shown in Supplementary Figure 1. Microarray profiles of JDM were retrieved from the Gene Expression Omnibus (GEO, http://www.ncbi.nlm.nih.gov/geo/) of the National Center for Biotechnology Information using the search terms of "juvenile dermatomyositis" restricted in the title. The datasets enrolled in this study must contain musclular specimens with three biological replicates at least. The "affy" package in R environment (version 3.6.1) was used to quantile normalize the expression within each dataset (Sasik et al. 2002). The 
82 corresponding platforms were applied to annotate each probe according to Entrez ID, and the 83 average expression value was calculated if several probes corresponded to the same Entrez ID. 84 (Supplementary Table 1). The "limma" R package was performed for identifying differentially 85 expressed genes (DEGs) between JDM samples and normal samples under cut-off criteria of false 86 discovery rate $($ FDR $)<0.05$ and $\mid \log 2$ fold change $\mid \geq 1$.

\section{Co-expression Network Construction}

The variance of each gene expression value was calculated and the genes with variance ranked in the top 25\% were selected for the construction of WGCNA (Langfelder \& Horvath 2008). The "WGNCA" package was used to construct the co-expression network. In detail, the function goodSamplesgenes was used to include the qualified genes and samples, followed by choosing an appropriate soft-thresholding power to construct the weighted adjacency matrix by the function pickSoftThreshold. The adjacency matrix was transformed into the topological matrix (TOM), and TOM-based dissimilarity (1-TOM) measure was used to cluster the genes using the flashClust function. Genes in the same module were highly interconnected. Then, phenotype (clinic traits) was imput into the co-expression network, and the following parameters were calculated: module eigengene (ME), gene significance (GS), and module membership (MM). ME represents the significant component in the principal component analysis for each gene module, and MM refers to the connectivity between genes and modules. GS was representative of correlation strength between gene expression and clinical traits, which was calculated by $\log 10$ transformation of the $P$-value $(\mathrm{GS}=\lg \mathrm{P})$ in the linear regression. Key modules were considered based on the criteria that the correlation coefficient $\geqslant 0.80$ and $P$-value $<0.05$.

Functional Enrichment Analysis

All genes in key modules were uploaded to the g:Profiler online (Reimand et al. 2007) database to perform Gene Ontology (GO) functional annotation (Ashburner et al. 2000) and the Kyoto encyclopedia of genes and genomes (KEGG) enrichment pathway analysis (Kanehisa \& Goto 2000). GO functional analysis consists of biological process (BP), cellular component (CC), and molecular function (MF). Analysis results were extracted under the condition of adjusted $P$ value $<0.05$. The top five terms were visualized if there were more than five terms.

\section{Selection and Validation of Hub Genes}

Genes with high correlation in candidate modules were defined as candidate hub genes. High connectivity was considered when the connectivity ranked in the top $2 \%$. Candidate hub gene met the absolute values of $\mathrm{MM}>0.80$ and GS $>0.20$. After identifying hub genes highly associated with clinical traits, the search tool for the retrieval of interacting genes (STRING) database was used to construct a protein-protein interaction (PPI) network for the candidate hub genes, and molecular complex detection (MCODE, a plugin in Cytoscape) was used to further select the real hub (Shannon et al. 2003; Szklarczyk et al. 2015). Genes with MCODE score $\geqslant 0$ in the PPI network were selected as the final hub genes. A separate dataset GSE11971 was used to validate the differential expression of the final hub genes.

\section{Related Small-Molecule Compounds Screening}

Connectivity map (CMap) database (https://portals.broadinstitute.org/cmap) was used to screen out small molecule compounds based on the real hub genes associated with JDM, because 
123

124

125

126

127

128

129

130

131

132

133

134

135

136

137

138

139

140

141

142

143

144

145

146

147

148

149

150

151

152

153

154

155

156

157

158

159

160

161

162

163

most compounds in this database are the United States Food and Drug Administration-approved drugs, (Lamb et al. 2006). First, real hub genes were divided into upregulated and downregulated groups. Next, these probe sets were used to query the CMap database based on the platform of the $\begin{array}{llllll}\text { Affymetrix Human } & \text { Genome } & \text { U133 } & \text { Plus }\end{array}$ (http://www.affymetrix.com/analysis/netaffx/index.affx ). Finally, enrichment scores representing similarity were calculated, ranging from -1 to 1 . Small molecules generated from up-regulated genes suggested therapeutic goals, while down-regulated genes predicted inhibitors of therapy for the disease. Potential compounds were selected based on connectivity score, $P$-value and correlation.

Statistical Analysis

Two-tailed Student's t-test was applied to the significance of differences between groups, and P-value less than 0.05 were considered as statistically significant. Statistical analyses were performed using Graphpad Prism 8.0.

\section{Results}

\section{Data Collection and Differentially Expressed Genes}

We employed two datasets on JDM muscular expression profiles. Dataset GSE3307 was used as the training set (Bakay et al. 2006). The original study enrolled 39 muscular biopsy samples, including 21 JDM patients and 18 healthy controls (HC). Dataset GSE11971, including nineteen JDM patients and four normal controls, was used as the validating set (Chen et al. 2008). The gene expression profiles of all tissue samples were analyzed based on the platform of the Affymetrix Human Genome U133 Plus 2.0 Array. A total of 2834 differentially expressed genes between JDM and $\mathrm{HC}$ were identified, including 1888 down-regulated genes and 946 up-regulated genes. The DEGs are listed in Supplementary Table 2.

Construction of a Weighted Co-expression Network and Identification of Key Modules

5103 genes whose variance ranked in the top $25 \%$ with $21 \mathrm{JDM}$ samples and 18 control samples in GSE3307 were used for WGCNA construction. The "WGCNA" R package was used for expression matrix of GSE3307, and soft-thresholding power $\beta$ value equal to 10 was selected to ensure a scale-free network with scale-free $\mathrm{R}^{2}$ equal to 0.90 (Supplementary Figure 2A-2C) (Langfelder \& Horvath 2008). A total of 13 modules were returned by WGCNA analysis (Figure $1 \mathrm{~A}-1 \mathrm{~B})$.

The interaction relationship of 12 modules was analyzed using network heatmap plots (Figure 1C). The division of all modules was highly independent from our analysis. The module eigengene dendrogram showed that 12 modules were divided into two clusters, and the adjacency heatmap of eigengene showed a similar result (Figure 2A). Based on the criteria that correlation coefficient $\geqslant 0.80, P$ value $<0.05$, blue, lightgreen and midnightblue modules were identified as key modules for further analysis (Figure 2B). Therefore, we selected the blue, lightgreen and midnightblue modules for subsequent analysis, to identify the relevance between key modules and the pathological state of JDM with substantial biological significance (Figure 2C-E).

\section{Functional and Pathway Enrichment Analysis}

GO and KEGG pathway enrichment was performed for all genes in the key modules to mine the biological functions associated with JDM. Biological process of GO analysis showed genes in 
164 the blue module were associated with generation of SRP-dependent cotranslational protein 165 targeting to membrane, cotranslational protein targeting to membrane, protein targeting to ER, 166 nuclear-transcribed mRNA catabolic process and establishment of protein localization to 167 endoplasmic reticulum; and that in the lightgreen module was relevant to response to type I 168 interferon, type I interferon signaling pathway, cellular response to type I interferon, defense 169 response to virus and response to virus. The top five pathways related to the midnightblue module 170 were cellular response to chemical stimulus, extracellular structure organization, extracellular 171 matrix organization, response to organic substance and cell motility (Figure 3A). Pathway 172 enrichment results of MF and CC in three key modules are presented in Figure 3B-3C. The results 173 of the KEGG pathway enrichment analysis in three modules are shown in Figure 3D.

174 Identification of Hub Genes

175

176

177

178

179

180

181

182

183

184

185

186

187

188

189

190

191

192

193

194

195

196

197

198

199

200

201

202

203

204

Based on the criteria that $\mathrm{MM}>0.80$ and $\mathrm{GS}>0.20$, a total of 45 DEGs with the high connectivity in key modules were screened as candidate hub genes. Then, a PPI network was constructed for candidate hub genes using Cytoscape, consisting of 42 nodes and 80 edges according to STRING database (Figure 4). We conducted molecular complex detection (MCODE) (a plugin in Cytoscape) analysis for 45 candidate hub genes, and 28 genes (blue $=15$, lightgreen $=11$, midnightblue $=1$ ) were considered hub genes according to the criteria of MCODE score $\geqslant$ 0 . Table 1 shows 28 hub genes in the three modules.

All hub genes were validated using JDM data from another GEO database (GSE11971). Because of the differences in microarray probes used in two data sets, boxplots were used to show the validation results for the final 22 hub genes (Supplementary Figure 3). We found that seven genes, SP110, SAMHD1, IFIT5, PLSCR1, IFI16, MX2 and CLIC1, were significantly upregulated in JDM compared to HC, while thirteen genes, COX5B, COX6A2, COX7C, NDUFA4, NDUFB4, MDH2, ATP5O, ATP5B, RPL21, TPI1, SLC25A3, VDAC1 and EIF4B, were significantly downregulated in JDM in comparison of HC. Figure 5 summarizes the cross-talk pathways involved in the pathogenesis of JDM by hub genes and literature(Miller et al. 2018; Thompson et al. 2017).

\section{Related Small-Molecule Compounds Screening}

The CMap database was used for small molecule drugs screening based on 20 real hub genes associated with JDM . Based on the criterion that the number of instances exceeds five and $P$ value less than 0.05, twelve small-molecule compounds were identified (Table 2). Among these compounds, acacetin, helveticoside, lanatoside $\mathrm{C}$, deferoxamine, famprofazone, tanespimycin and LY-294002 may perturb the development of JDM, while betonicine, felodipine, valproic acid, and sirolimus might provide potentially therapeutic goals for JDM.

\section{Discussion}

In this study, we used WGCNA to construct a co-expression network, detect key gene modules and identify hub genes in JDM for the first time. Our research provides some potential biomarkers or molecular targets for JDM through the Cmap database. We found that three modules highly correlated with JDM. The expression of 28 genes in these three modules showed significant changes in patients with JDM compared to control individuals in the training period, 
205

206

207

208

209

210

211

212

213

214

215

216

217

218

219

220

221

222

223

224

225

226

227

228

229

230

231

232

233

234

235

236

237

238

239

240

241

242

243

244

245

246

247

248

249

and 20 genes were validated as the real hub genes in the GSE11971 dataset, including the downregulation of NADH dehydrogenase, ATP synthase and cytochrome c oxidase and upregulation of IFN-stimulated genes. However, few of them were identified as biomarkers or crucial genes in JDM yet.

Functional enrichment analysis indicated that type I interferon signaling and various virus infection pathways were strengthened in JDM compared to $\mathrm{HC}$, which is consistent with findings of previous studies (Moneta et al. 2019; Piper et al. 2018). IFIT5, IFI16 and MX2, interferonstimulated genes, both nuclear transcriptional factors, were found to be upregulated in other autoimmune diseases but not in JDM (Wang et al. 2019b; Zhang \& Xu 2019). In the present study, we found that interferon-stimulated genes were significantly upregulated in JDM patients, as well as the so-called "interferon signature", demonstrating a possible mechanism that viral mimics or other stimuli may play a crucial role in the pathogenesis of JDM. Viral mimics are thought to participate in the pathogenesis of JDM (Musumeci \& Castrogiovanni 2018) and other autoimmune diseases (Christen et al. 2004; Sellami et al. 2019), consistent with the notion that JDM patients have higher rates of viral infections (Tansley et al. 2013; Zheng et al. 2019). This may suggest that the prevention of certain viral infections would decrease the incidence of autoimmunity by inhibiting self-antigenic mimics. NADH dehydrogenase (NDUFA4 and NDUFB4), ATP synthase (ATP5O and ATP5B) and cytochrome c oxidase (COX) family (COX5B, COX6A2 and COX7C) are crucial molecules involved in the oxidative phosphorylation in mitochondrial metabolism, and the decreased levels of these molecules suggested a crucial role of impaired mitochondrial phosphorylation and lower oxidative capacity in the pathogenesis of JDM, accounting for the extremity weakness in JDM patients.

Hypoxia caused by suppressed oxidative phosphorylation induces changes in reactive oxygen species (ROS) generation, whereby severe hypoxia in skeletal muscle results in elevated $\mathrm{H}_{2} \mathrm{O}_{2}$ generation. ROS accumulation produced by mitochondrial dysfunctions, in turn, drives type I interferon responses and muscle inflammation, and may thereby self-sustain the disease process (Wang et al. 2019a). Similar to other autoimmune diseases, high-dose glucocorticoids, used alone or in combination with immunosuppressive agents are routine treatment for JDM patients wheras some refractory patients may develop functional limitations. It has been suggested that refractory JDM patients, have lower maximal oxygen uptake(Drinkard et al. 2003; Hicks et al. 2002) than do healthy children and with children with juvenile dermatomyositis in remission(Takken et al. 2008), suggesting that mitochrondrial dysfuction may contribute to the severity of JDM. Current concepts on the therapy of muscle weakness in JDM focus on induction of partial recovery and exposure to serious adverse events (including muscular toxicity). Our data suggest a novel therapeutic perspective for JDM by protecting mitochondria from dysfunction.

Bioinformatics combined human and material resources to develop more efficient tools with lower error rates (Irizarry et al. 2003). WGCNA is an efficient approach to construct co-expressed modules and hub genes in several diseases. Previous studies using microarray expression profiles from adult-onset DM patients showed that IFN-stimulated genes were upregulated (i.e. MX2, STAT1 and OAS3), suggesting that the IFN signature overlapped the pathogenesis both in adult and juvenile DM . Nevertheless, mechanisms linked to hypoxia are less prevalent in adult-onset DM, suggesting mitochondrial dysfunctions contribute more to juvenile-onset DM rather than adult-onset DM.

We used the CMap database to predict several kinds of small-molecule compounds with promising capacity as therapeutic goals or inhibitors on treatment for JDM. No evidence has

Peer) reviewing PDF | (2019:10:42389:2:0:NEW 13 Jan 2020) 
250

251

252

253

254

255

256

257

258

259

260

261

262

263

264

265

266

267

268

269

270

271

272

273

274

275

276

277

278

279

280

281

282

283

284

285

286

287

288

289

290

291

292

293

demonstrated the direct association between these compounds and JDM, while they hinted indirect link to JDM, according to the literatue. Among these compounds, acacetin, helveticoside, lanatoside C, deferoxamine, famprofazone, tanespimycin and LY-294002 showed negative enrichment scores and thus may have the potential to perturb the development of JDM, while betonicine, felodipine, valproic acid, and sirolimus showed positive enrichment scores and might provide potentially therapeutic goals for JDM. Acacetin, an inhibitor of lipopolysaccharideinduced inflammation, can promote the expansion of Treg cells and supress the differentiation of Th17 cells in a dose-dependent manner in collagen-induced arthritis (Liu et al. 2018). Helveticoside can regulate metabolism and signaling processes as a biologically active component, but little is known in inflammatory reactions (Kim et al. 2015). The iron chelator deferoxamine was shown to reduce mitochondrial oxidative stress in a transient cerebral ischemia model as well as the release of pro-inflammatory molecules including matrix metalloproteinase- 9 and hypoxia inducible factor-1 (Im et al. 2012). LY294002, a kind of PI3K inhibitor, has potential against experimental autoimmune myocarditis (Liu et al. 2016). The heat-shock protein 90 inhibitor tanespimycin has been shown to inhibit cutaneous inflammation in experimental epidermolysis bullosa acquisita (Tukaj et al. 2017) and other experimental autoimmune models (Dello Russo et al. 2006). Felodipine, commonly used to treat hypertension and angin, has been evidenced to inhibit oxidative stress and inflammation in endothelial cells, which is consistent with our results (Qi et al. 2017). Valproic acid is a histone deacetylase inhibitor (HDACI), can suppress the inflammatory responses mediated by cytokines, oxidative stress molecules (ROS, NO), activating receptors (NK, T $\gamma \delta$, and cytotoxic lymphocytes), perforin, granzyme, costimulatory molecules, and autoantibodies(Soria-Castro et al. 2019). Sirolimus can restore immune balance in rheumatoid arthritis patients by expanding the pool of circulating Treg cells(Niu et al. 2019). Our results based on the CMap database might provide hints as to future therapy for JDM; nevertheless, studies in vitro and in vivo are necessary.

This study has some limitations. First, this is retrospective, with all data in this study being retrieved from a public database. A multicenter, prospective study is needed to evaluate the significance of these hub genes in terms of long-term outcomes and possible applications of molecular drugs for therapy. Second, experiments in vivo and in vitro are necessary to interpret potential mechanisms of real hub genes and small-molecule compounds for future clinical translation. Third, clinical traits cannot correlate with gene modules when performing WGCNA because of lack of clinical trait data in these GEO datasets.

\section{Conclusions}

Based on weighted gene co-expression analysis, three key modules and 20 real key genes associated with the pathological state of JDM were identified, suggesting pivotal roles of mitochondrial dysfunction and the interferon signature in JDM. This analysis provides several candidate small-molecule compounds for use as targeted therapy of JDM.

\section{Acknowledgements}

This work was supported by Chinese National Key Technology R\&D Program of Ministry of Science and Technology (2017YFC0907604), National Science and Technology Major Project of the Ministry of Science and Technology of China (2019ZX09734001-002-004), Medical and health science and technology innovation project of Chinese Academy of Medical Sciences (2019-I2M-2-008), National Natural Science Foundation of China (81601430, 81471615).

Peer] reviewing PDF | (2019:10:42389:2:0:NEW 13 Jan 2020) 
We would like to acknowledge the GEO, g:Profiler, STRING and CMap databases for free use.

\section{References}

312

Arabshahi B, Silverman RA, Jones OY, and Rider LG. 2012. Abatacept and sodium thiosulfate for treatment of recalcitrant juvenile dermatomyositis complicated by ulceration and calcinosis. J Pediatr 160:520-522. 10.1016/j.jpeds.2011.11.057

Ashburner M, Ball CA, Blake JA, Botstein D, Butler H, Cherry JM, Davis AP, Dolinski K, Dwight SS, Eppig JT, Harris MA, Hill DP, Issel-Tarver L, Kasarskis A, Lewis S, Matese JC, Richardson JE, Ringwald M, Rubin GM, and Sherlock G. 2000. Gene ontology: tool for the unification of biology. The Gene Ontology Consortium. Nat Genet 25:25-29. $10.1038 / 75556$

Bakay M, Wang Z, Melcon G, Schiltz L, Xuan J, Zhao P, Sartorelli V, Seo J, Pegoraro E, Angelini C, Shneiderman B, Escolar D, Chen YW, Winokur ST, Pachman LM, Fan C, Mandler R, Nevo Y, Gordon E, Zhu Y, Dong Y, Wang Y, and Hoffman EP. 2006. Nuclear envelope dystrophies show a transcriptional fingerprint suggesting disruption of RbMyoD pathways in muscle regeneration. Brain 129:996-1013. 10.1093/brain/awl023

Chen YW, Shi R, Geraci N, Shrestha S, Gordish-Dressman H, and Pachman LM. 2008. Duration of chronic inflammation alters gene expression in muscle from untreated girls with juvenile dermatomyositis. BMC Immunol 9:43. 10.1186/1471-2172-9-43

Christen U, Edelmann KH, McGavern DB, Wolfe T, Coon B, Teague MK, Miller SD, Oldstone MB, and von Herrath MG. 2004. A viral epitope that mimics a self antigen can accelerate but not initiate autoimmune diabetes. J Clin Invest 114:1290-1298. 10.1172/jci22557

Crowe WE, Bove KE, Levinson JE, and Hilton PK. 1982. Clinical and pathogenetic implications of histopathology in childhood polydermatomyositis. Arthritis Rheum 25:126-139. 10.1002/art.1780250203 
335

336

337

338

339

340

341

342

343

344

345

346

347

348

349

350

351

352

353

354

355

356

357

358

359

360

361

362

363

364

365

366

367

368

369

370

371

372

373

374

375

376

377

378

379

380

381

382

383

384

385

De Paepe B. 2017. Vascular changes and perifascicular muscle fiber damage in dermatomyositis: another question of the chicken or the egg that is on our mind. Ann Transl Med 5:22. 10.21037/atm.2016.12.68

Dello Russo C, Polak PE, Mercado PR, Spagnolo A, Sharp A, Murphy P, Kamal A, Burrows FJ, Fritz LC, and Feinstein DL. 2006. The heat-shock protein 90 inhibitor 17-allylamino-17demethoxygeldanamycin suppresses glial inflammatory responses and ameliorates experimental autoimmune encephalomyelitis. J Neurochem 99:1351-1362. 10.1111/j.1471-4159.2006.04221.x

Drinkard BE, Hicks J, Danoff J, and Rider LG. 2003. Fitness as a determinant of the oxygen uptake/work rate slope in healthy children and children with inflammatory myopathy. Can J Appl Physiol 28:888-897. 10.1139/h03-063

Feldman BM, Rider LG, Reed AM, and Pachman LM. 2008. Juvenile dermatomyositis and other idiopathic inflammatory myopathies of childhood. Lancet 371:2201-2212. 10.1016/s0140-6736(08)60955-1

Gitiaux C, De Antonio M, Aouizerate J, Gherardi RK, Guilbert T, Barnerias C, Bodemer C, Brochard-Payet K, Quartier P, Musset L, Chazaud B, Desguerre I, and Bader-Meunier B. 2016. Vasculopathy-related clinical and pathological features are associated with severe juvenile dermatomyositis. Rheumatology (Oxford) 55:470-479. 10.1093/rheumatology/kev359

Greenberg SA. 2010. Dermatomyositis and type 1 interferons. Curr Rheumatol Rep 12:198-203. 10.1007/s11926-010-0101-6

Hicks JE, Drinkard B, Summers RM, and Rider LG. 2002. Decreased aerobic capacity in children with juvenile dermatomyositis. Arthritis Rheum 47:118-123. 10.1002/art.10237

Hoeltzel MF, Oberle EJ, Robinson AB, Agarwal A, and Rider LG. 2014. The presentation, assessment, pathogenesis, and treatment of calcinosis in juvenile dermatomyositis. Curr Rheumatol Rep 16:467. 10.1007/s11926-014-0467-y

Im DS, Jeon JW, Lee JS, Won SJ, Cho SI, Lee YB, and Gwag BJ. 2012. Role of the NMDA receptor and iron on free radical production and brain damage following transient middle cerebral artery occlusion. Brain Res 1455:114-123. 10.1016/j.brainres.2012.03.025

Irizarry RA, Hobbs B, Collin F, Beazer-Barclay YD, Antonellis KJ, Scherf U, and Speed TP. 2003. Exploration, normalization, and summaries of high density oligonucleotide array probe level data. Biostatistics 4:249-264. 10.1093/biostatistics/4.2.249

Kanehisa M, and Goto S. 2000. KEGG: kyoto encyclopedia of genes and genomes. Nucleic Acids Res 28:27-30. 10.1093/nar/28.1.27

Kim BY, Lee J, and Kim NS. 2015. Helveticoside is a biologically active component of the seed extract of Descurainia sophia and induces reciprocal gene regulation in A549 human lung cancer cells. BMC Genomics 16:713. 10.1186/s12864-015-1918-1

Lamb J, Crawford ED, Peck D, Modell JW, Blat IC, Wrobel MJ, Lerner J, Brunet JP, Subramanian A, Ross KN, Reich M, Hieronymus H, Wei G, Armstrong SA, Haggarty SJ, Clemons PA, Wei R, Carr SA, Lander ES, and Golub TR. 2006. The Connectivity Map: using gene-expression signatures to connect small molecules, genes, and disease. Science 313:1929-1935. 10.1126/science.1132939

Langfelder $P$, and Horvath S. 2008. WGCNA: an R package for weighted correlation network analysis. BMC Bioinformatics 9:559. 10.1186/1471-2105-9-559

Li J, and Zhou Z. 2019. Calcinosis in Juvenile Dermatomyositis. N Engl J Med 381:e31. 10.1056/NEJMicm1809669

Lindsley C, Laxer RM, Cassidy JT, and Petty R. 1995. Textbook of Pediatric Rheumatology [M]. Philadelphia: Elsevier Saunders:539-546. 10.1016/C2009-0-42830-7

Liu HS, Zhang J, Guo JL, Lin CY, and Wang ZW. 2016. Phosphoinositide 3-kinase inhibitor LY294002 ameliorates the severity of myosin-induced myocarditis in mice. Curr Res Transl Med 64:21-27. 10.1016/j.retram.2016.01.012

Peer) reviewing PDF | (2019:10:42389:2:0:NEW 13 Jan 2020) 
386

387

388

389

390

391

392

393

394

395

396

397

398

399

400

401

402

403

404

405

406

407

408

409

410

411

412

413

414

415

416

417

418

419

420

421

422

423

424

425

426

427

428

429

430

431

432

433

434

435

Liu L, Yang J, Zu B, Wang J, Sheng K, Zhao L, and Xu W. 2018. Acacetin regulated the reciprocal differentiation of Th17 cells and Treg cells and mitigated the symptoms of collagen-induced arthritis in mice. Scand J Immunol 88:e12712. 10.1111/sji.12712

Miller FW, Lamb JA, Schmidt J, and Nagaraju K. 2018. Risk factors and disease mechanisms in myositis. Nat Rev Rheumatol 14:255-268. 10.1038/nrrheum.2018.48

Moneta GM, Pires Marafon D, and Marasco E. 2019. Muscle Expression of Type I and Type II Interferons Is Increased in Juvenile Dermatomyositis and Related to Clinical and Histologic Features. 71:1011-1021. 10.1002/art.40800

Musumeci G, and Castrogiovanni P. 2018. Expression of the OAS Gene Family Is Highly Modulated in Subjects Affected by Juvenile Dermatomyositis, Resembling an Immune Response to a dsRNA Virus Infection. 19. 10.3390/ijms19092786

Niu HQ, Li ZH, Zhao WP, Zhao XC, Zhang C, Luo J, Lu XC, Gao C, Wang CH, and Li XF. 2019. Sirolimus selectively increases circulating Treg cell numbers and restores the Th17/Treg balance in rheumatoid arthritis patients with low disease activity or in DAS28 remission who previously received conventional disease-modifying anti-rheumatic drugs. Clin Exp Rheumatol.

Nordal E, Pistorio A, Rygg M, Giancane G, Maghnie M, Di lorgi N, Flemming K, Hofer M, MeloGomes JA, Bica B, Brunner J, Dannecker G, Gerloni V, Harjacek M, Huppertz HI, Pratsidou-Gertsi P, Nielsen S, Stanevicha V, Ten Cate R, Vougiouka O, Pastore S, Simonini G, Ravelli A, Martini A, and Ruperto N. 2019. Growth and puberty in juvenile dermatomyositis: a longitudinal cohort study. Arthritis Care Res (Hoboken). 10.1002/acr.24065

Piper CJM, Wilkinson MGL, Deakin CT, Otto GW, Dowle S, Duurland CL, Adams S, Marasco E, Rosser EC, Radziszewska A, Carsetti R, loannou Y, Beales PL, Kelberman D, Isenberg DA, Mauri C, Nistala K, and Wedderburn LR. 2018. CD19(+)CD24(hi)CD38(hi) B Cells Are Expanded in Juvenile Dermatomyositis and Exhibit a Pro-Inflammatory Phenotype After Activation Through Toll-Like Receptor 7 and Interferon-alpha. Front Immunol 9:1372. 10.3389/fimmu.2018.01372

Qi J, Zheng JB, Ai WT, Yao XW, Liang L, Cheng G, Shou XL, and Sun CF. 2017. Felodipine inhibits ox-LDL-induced reactive oxygen species production and inflammation in human umbilical vein endothelial cells. Mol Med Rep 16:4871-4878. 10.3892/mmr.2017.7181

Ravelli A, Trail L, Ferrari C, Ruperto N, Pistorio A, Pilkington C, Maillard S, Oliveira SK, Sztajnbok F, Cuttica R, Beltramelli M, Corona F, Katsicas MM, Russo R, Ferriani V, Burgos-Vargas R, Magni-Manzoni S, Solis-Valleoj E, Bandeira M, Zulian F, Baca V, Cortis E, Falcini F, Alessio M, Alpigiani MG, Gerloni V, Saad-Magalhaes C, Podda R, Silva CA, Lepore L, Felici E, Rossi F, Sala E, and Martini A. 2010. Long-term outcome and prognostic factors of juvenile dermatomyositis: a multinational, multicenter study of 490 patients. Arthritis Care Res (Hoboken) 62:63-72. 10.1002/acr.20015

Reimand J, Kull M, Peterson H, Hansen J, and Vilo J. 2007. g:Profiler--a web-based toolset for functional profiling of gene lists from large-scale experiments. Nucleic Acids Res 35:W193-200. 10.1093/nar/gkm226

Sasik R, Calvo E, and Corbeil J. 2002. Statistical analysis of high-density oligonucleotide arrays: a multiplicative noise model. Bioinformatics 18:1633-1640.

10.1093/bioinformatics/18.12.1633

Sellami M, Saidane O, Mahmoud I, Tekaya AB, Tekaya R, and Abdelmoula L. 2019. Etiological features of liver involvement in rheumatoid arthritis. Curr Rheumatol Rev. 10.2174/1573397115666191007121605

Shannon P, Markiel A, Ozier O, Baliga NS, Wang JT, Ramage D, Amin N, Schwikowski B, and Ideker T. 2003. Cytoscape: a software environment for integrated models of biomolecular interaction networks. Genome Res 13:2498-2504. 10.1101/gr.1239303

Peer) reviewing PDF | (2019:10:42389:2:0:NEW 13 Jan 2020) 
436

437

438

439

440

441

442

443

444

445

446

447

448

449

450

451

452

453

454

455

456

457

458

459

460

461

462

463

464

465

466

467

468

469

470

471

472

473

474

475

476

477

478

479

480

481

482

483

Soria-Castro R, Schcolnik-Cabrera A, Rodriguez-Lopez G, Campillo-Navarro M, Puebla-Osorio N, Estrada-Parra S, Estrada-Garcia I, Chacon-Salinas R, and Chavez-Blanco AD. 2019. Exploring the Drug Repurposing Versatility of Valproic Acid as a Multifunctional Regulator of Innate and Adaptive Immune Cells. J Immunol Res 2019:9678098. $10.1155 / 2019 / 9678098$

Szklarczyk D, Franceschini A, Wyder S, Forslund K, Heller D, Huerta-Cepas J, Simonovic M, Roth A, Santos A, Tsafou KP, Kuhn M, Bork P, Jensen LJ, and von Mering C. 2015. STRING v10: protein-protein interaction networks, integrated over the tree of life. Nucleic Acids Res 43:D447-452. 10.1093/nar/gku1003

Takken T, van der Net J, Engelbert RH, Pater S, and Helders PJ. 2008. Responsiveness of exercise parameters in children with inflammatory myositis. Arthritis Rheum 59:59-64. 10.1002/art.23250

Tansley SL, McHugh NJ, and Wedderburn LR. 2013. Adult and juvenile dermatomyositis: are the distinct clinical features explained by our current understanding of serological subgroups and pathogenic mechanisms? Arthritis Res Ther 15:211. 10.1186/ar4198

Thompson C, Piguet V, and Choy E. 2017. The pathogenesis of dermatomyositis. Br J Dermatol. 10.1111/bjd. 15607

Tukaj S, Bieber K, Kleszczynski K, Witte M, Cames R, Kalies K, Zillikens D, Ludwig RJ, Fischer TW, and Kasperkiewicz M. 2017. Topically Applied Hsp90 Blocker 17AAG Inhibits Autoantibody-Mediated Blister-Inducing Cutaneous Inflammation. J Invest Dermatol 137:341-349. 10.1016/j.jid.2016.08.032

Wang HW, Zhang Y, Tan PP, Jia LS, Chen Y, and Zhou BH. 2019a. Mitochondrial respiratory chain dysfunction mediated by ROS is a primary point of fluoride-induced damage in Hepa1-6 cells. Environ Pollut 255:113359. 10.1016/j.envpol.2019.113359

Wang X, Zhang Z, Zhao W, Li Z, Yang G, Liu P, Jia J, Shi H, Liu M, Liu T, Gu J, Wan L, Teng J, Liu H, Cheng X, Ye J, Su Y, Sun Y, Gong W, Yang C, and Hu Q. 2019b.

Cytomegalovirus Infection May Trigger Adult-Onset Still's Disease Onset or Relapses. J Cell Biochem 10:898. 10.1002/jcb.29001

10.3389/fimmu.2019.00898

Zhang L, and Xu P. 2019. Identification of differentially expressed genes in primary Sjogren's syndrome. 120:17368-17377. 10.1002/hep.30897

$10.1002 / j \mathrm{jcb} .29001$

Zhao W, Langfelder P, Fuller T, Dong J, Li A, and Hovarth S. 2010. Weighted gene coexpression network analysis: state of the art. J Biopharm Stat 20:281-300. 10.1080/10543400903572753

Zheng Q, Zhu K, Gao CN, Xu YP, and Lu MP. 2019. Prevalence of Epstein-Barr virus infection and characteristics of lymphocyte subsets in newly onset juvenile dermatomyositis. 10.1007/s12519-019-00314-7

Figure legends

Figure 1. Construction of WGCNA modules. (A) Dendrogram of module eigengenes based on dissimilarity measure (1-TOM); (B) Cluster dendrogram of the genes with variance ranked in the top 25\% in GSE3307 dataset. Each color represents one module. The blue module contained the most genes $(n=1549)$, followed by lightgreen $(n=941)$, black $(n=428)$, darkgrey $(n=367)$, midnightblue $(n=280)$, darkred $(n=251)$, magenta $(n=211)$, purple $(n=196)$, salmon $(n=172)$, lightcyan $(n=169)$, darkgreen $(n=100)$, and darkturquoise $(n=96)$ modules. The 343 genes that could not be incorporated into 
484

485

486

487

488

489

490

491

492

493

494

495

496

497

498

499

500

501

502

503

504

505

506

507

508

509

510

511

512

513

514

515

516

517

518

519

520

521

522

any modules belonged to the grey module, and were removed in the subsequent analysis; (C) Network heatmap plots of genes selected for WGCNA construction. The depth of yellow in the middle of the figure indicated the degree of correlation between pair-wise genes.

Figure 2. Relationship between modules and clinical traits. (A) Module eigengene dendrogram and Adjacency heatmap. (B) Heatmap of the module-trait relationships. (CE) Scatter plots of module eigengenes in blue(C), lightgreen $(\mathbf{D})$ and midnightblue $(\mathbf{E})$ modules.

Figure 3. Top 5 terms of GO and KEGG pathway enrichment analysis in blue, lightgreen and midnightblue modules. The horizontal axis represents the gene ratio, and the vertical axis indicates the term of GO/KEGG signaling pathway, and the change of color from blue to red indicates the change of significance from low to high. (A) Biological function; (B) cellular component; (C) molecular function; (D) KEGG enrichment pathway.

Figure 4. Protein-protein interaction network of 45 hub genes. Different shapes represent various clusters constructed by molecular complex detection (MCODE). i.e. ellipses represent cluster 1 and triangles represent cluster 2 . The color changes from dark brown to light brown indicate the MCODE score changes from high to low.

Figure 5. Cross-talk pathways involved in JDM interfered by hub genes and reference. Enviromental factors (such as the invasion of virus) stimulate receptors to pathogenassociated molecular patterns (PAMPs) or cytokines on skeletal muscle cells and vascular endothelial cells, followed by the activation of DNA-cGAS-STING axis or RNAMDA5/RIG-I-MAVS axis, thereby inducing the transcription of interferon-stimulated genes and other genes by phosphorylating IRF3/7, ultimately leading to inflammatory infiltration in blood vessels as well as skeletal muscle weakness. The dysfunction of metabolic signallings through cytokine receptors (for example, the type I interferon receptor (IFNAR) and/or IL-1 receptor (IL-1R)) activated oxidative phosphorylation in mitochondria, leading to the accumulation of reactive oxygen species (ROS), potentially resulting in energy-generating deficits in skeletal muscles. The hub genes involved in the pathogenesis of JDM are as follows: IFN-stimulated genes: IFIT5, IFI16 and MX2; Cytochrome c oxidase: COX5B, COX6A2 and COX7C; NADH dehydrogenase: NDUFA4 and NDUFB4; ATP synthase: ATP5B and ATP5O.

Supplementary Figure 1. The flowchart of this study.

Supplementary Figure 2. Clustering dendrogram of all samples and determination of soft-thresholding power. (A) Sample clustering was conducted to detect outliers. All samples are located in the clusters and pass the cutoff thresholds. (B) Analysis of the scale-free fit index for various soft-thresholding powers $(\beta)$. (C) Analysis of the mean connectivity for various soft-thresholding powers.

Supplementary Figure 3. Boxplots of 22 hub genes. (A) ATP5O, ATP5B, NDUFV2, COX7C, COX5B, NDUFB4 and COX6A2; (B) NDUFA4, VDAC1, SLC25A3, MDH2, 
523 TPI1, RPL21, MRPS7 and MX2; (C) SAMHD1, EIF4B, IFIT5, SP110, IFI16, PLSCR1, 524 and CLIC1. 


\section{Figure 1}

Figure 1

Construction of WGCNA modules. (A) Dendrogram of module eigengenes based on

dissimilarity measure (1-TOM); (B) Cluster dendrogram of the genes with variance ranked in the top 25\% in GSE3307 dataset. Each color represents one module; (C) Network heatmap plots of genes selected for WGCNA construction. The depth of yellow in the middle of the figure indicated the degree of correlation between pair-wise genes.

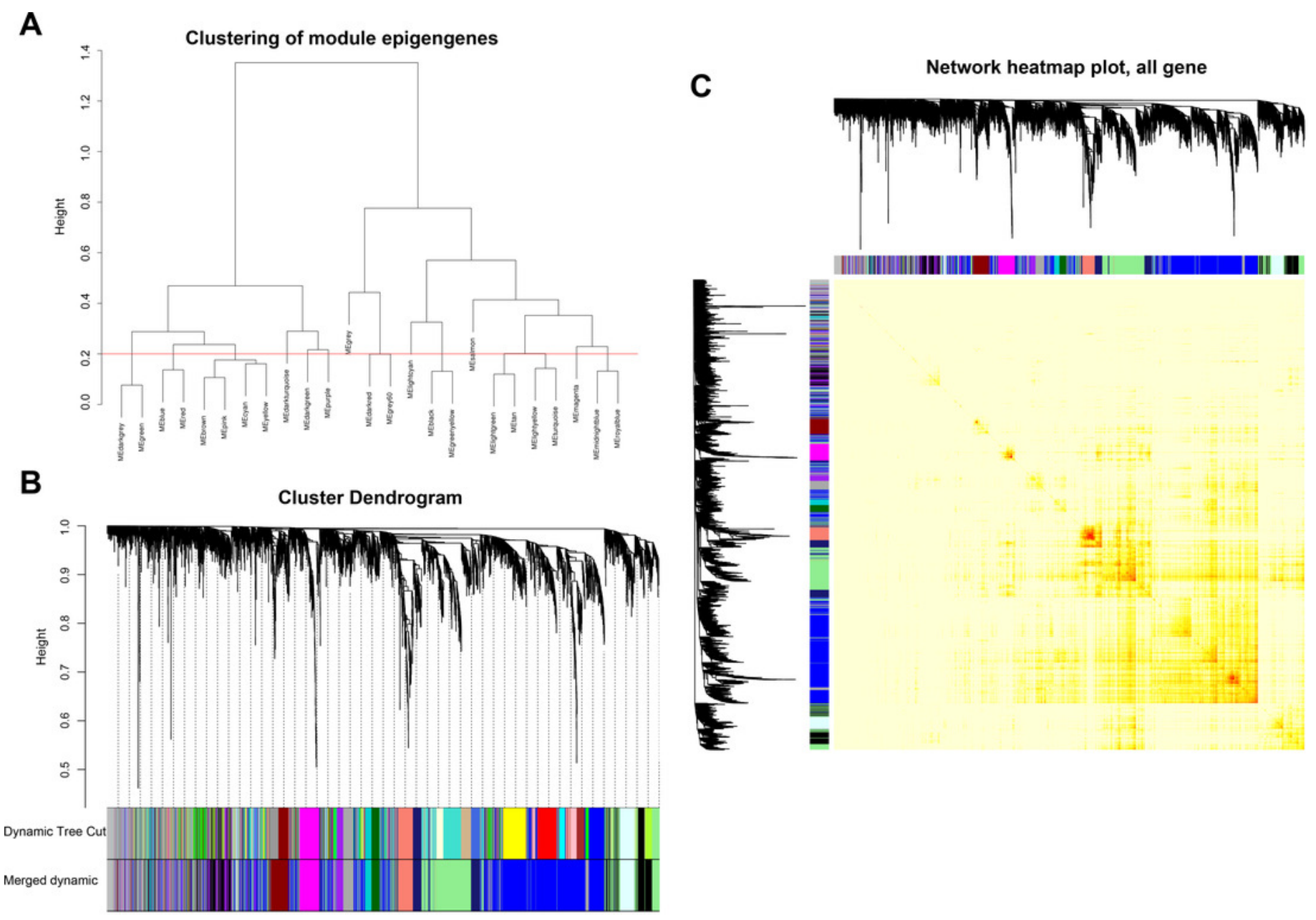


Figure 2

Figure 2

Relationship between modules and clinical traits. (A) Module eigengene dendrogram and Adjacency heatmap. (B) Heatmap of the module-trait relationships. (C-E) Scatter plots of module eigengenes in blue(C), lightgreen (D) and midnightblue (E) modules.

A
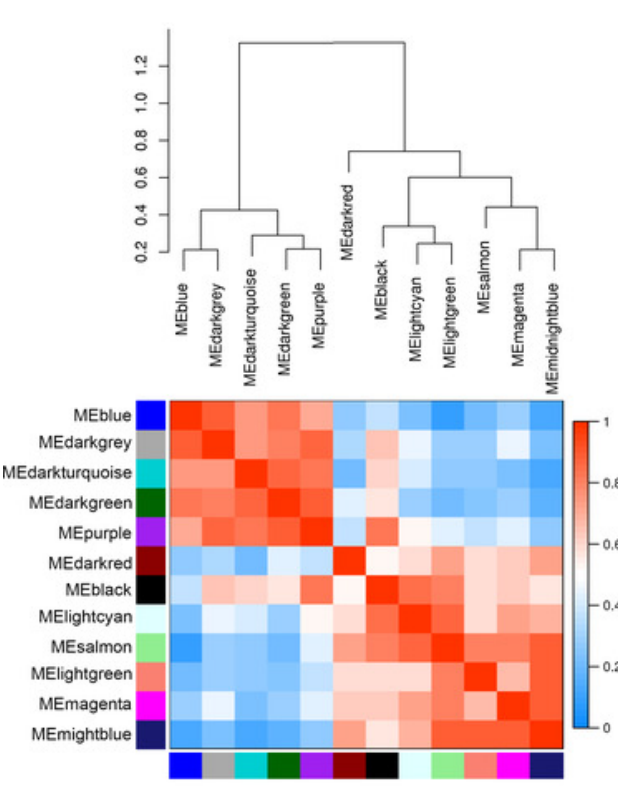

B
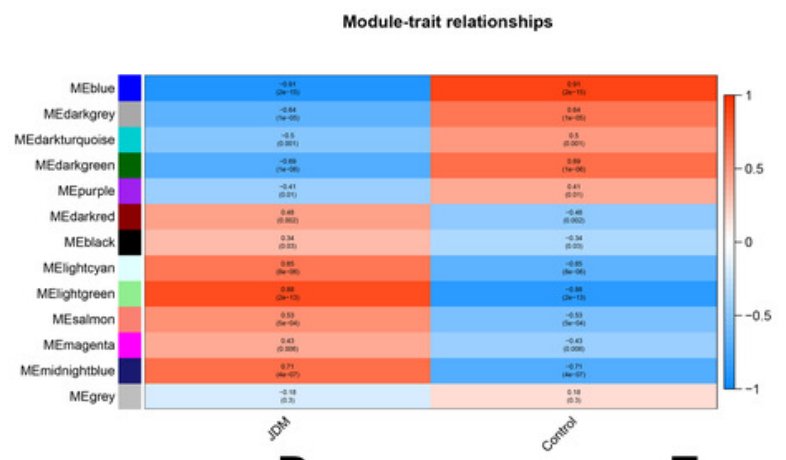
$\mathbf{C}$ Module membership vs. gene significance $\mathbf{D}$ Module membership vs. gene significance $\mathbf{E}$
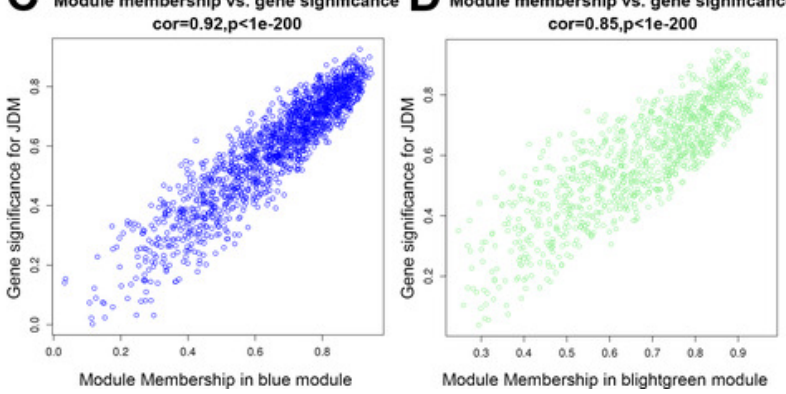

Module membership vs. gene significance

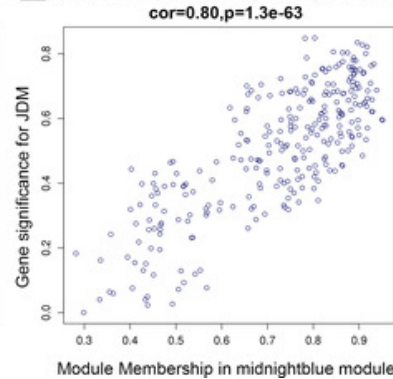


Figure 3

Figure 3

Top 5 terms of GO and KEGG pathway enrichment analysis in blue, lightgreen and midnightblue modules. The horizontal axis represents the gene ratio, and the vertical axis indicates the term of GO/KEGG signaling pathway, and the change of color from blue to red indicates the change of significance from low to high. (A) Biological function; (B) cellular component; (C) molecular function; (D) KEGG enrichment pathway.
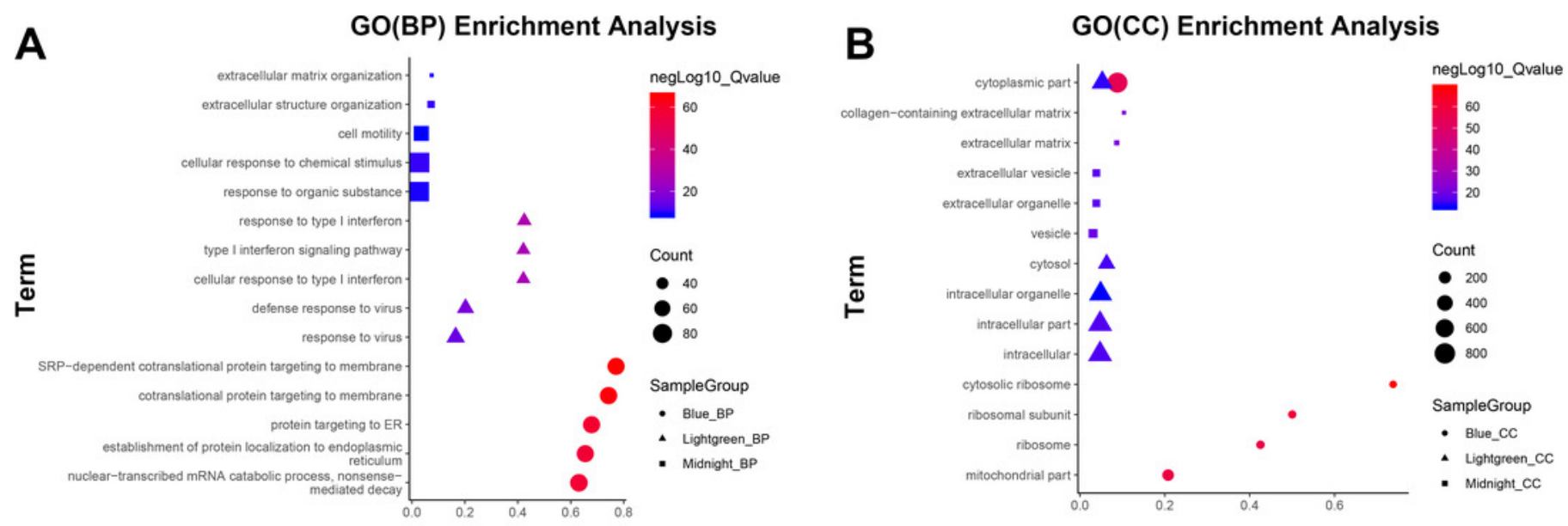

C GO(MF) Enrichment Analysis

D

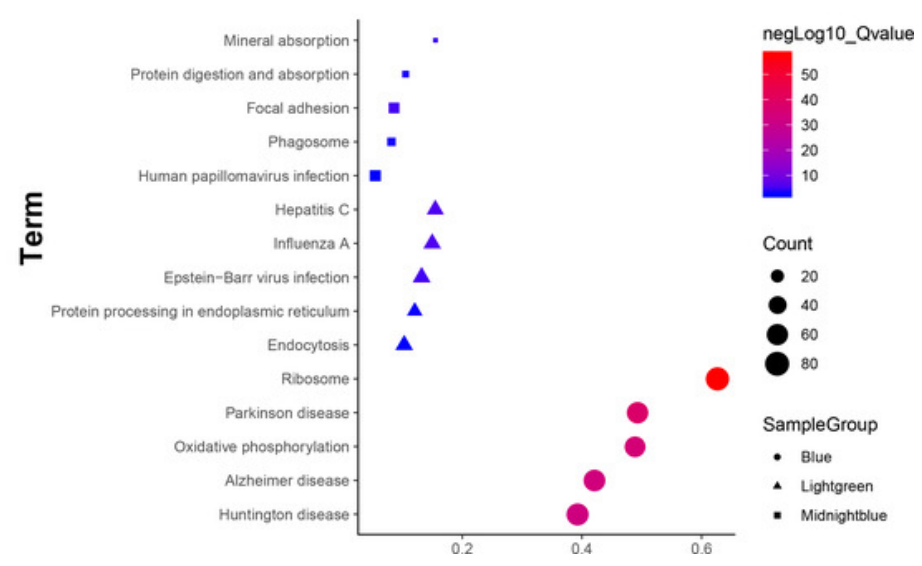


Figure 4

Figure 4

Protein-protein interaction network of 45 hub genes. Different shapes represent various clusters constructed by molecular complex detection (MCODE). i.e. ellipses represent cluster 1 and triangles represent cluster 2 . The color changes from dark brown to light brown indicate the MCODE score changes from high to low.
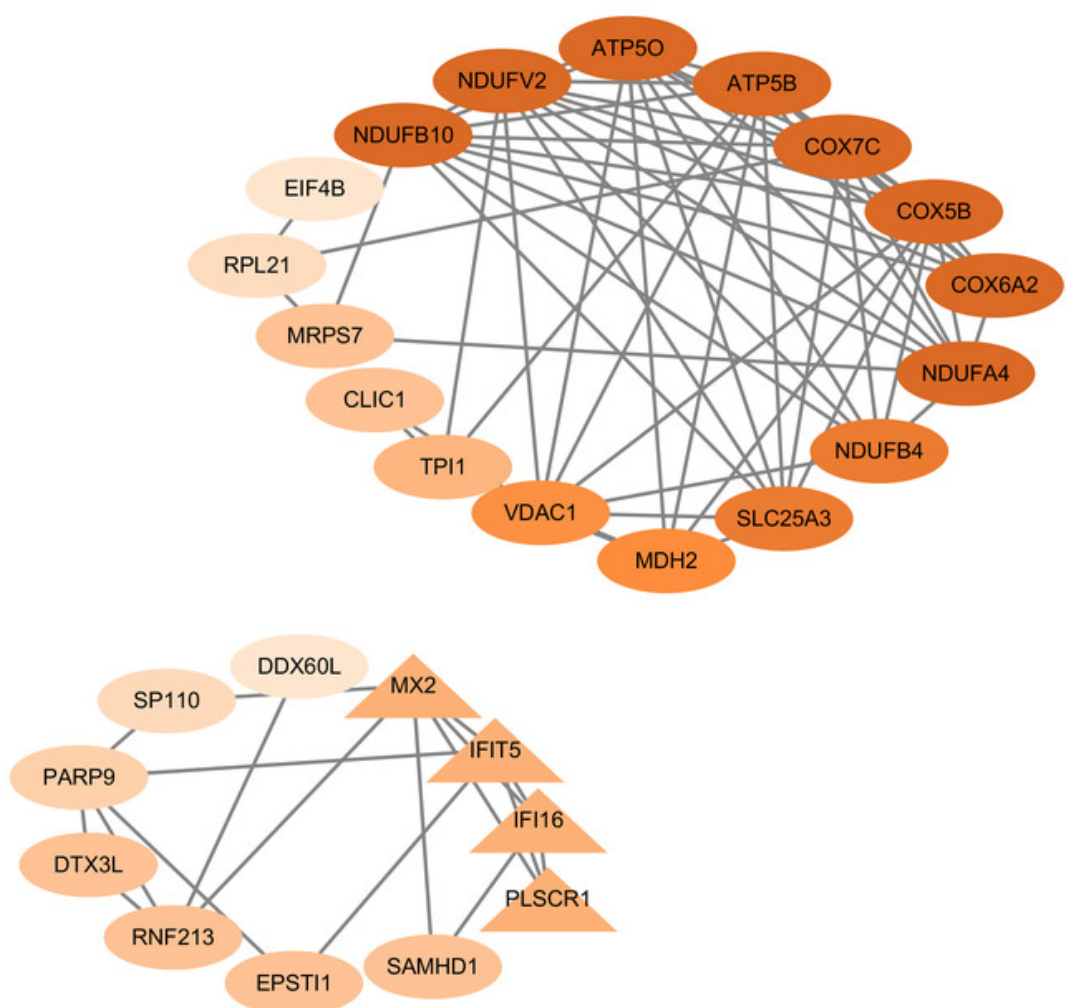


\section{Figure 5}

Figure 5

Cross-talk pathways involved in JDM interfered by hub genes and reference. Enviromental factors (such as the invasion of virus) stimulate receptors to pathogen-associated molecular patterns (PAMPs) or cytokines on skeletal muscle cells and vascular endothelial cells, followed by the activation of DNA-cGAS-STING axis or RNA-MDA5/RIG-I-MAVS axis, thereby inducing the transcription of interferon-stimulated genes and other genes by phosphorylating IRF3/7, ultimately leading to inflammatory infiltration in blood vessels as well as skeletal muscle weakness. The dysfunction of metabolic signallings through cytokine receptors (for example, the type I interferon receptor (IFNAR) and/or IL-1 receptor (IL-1R)) activated oxidative phosphorylation in mitochondria, leading to the accumulation of reactive oxygen species (ROS), potentially resulting in energy-generating deficits in skeletal muscles. The hub genes involved in the pathogenesis of JDM are as follows: IFN-stimulated genes: IFIT5, IFI16 and MX2; Cytochrome c oxidase: COX5B, COX6A2 and COX7C; NADH dehydrogenase: NDUFA4 and NDUFB4 ; ATP synthase: ATP5B and ATP50. 

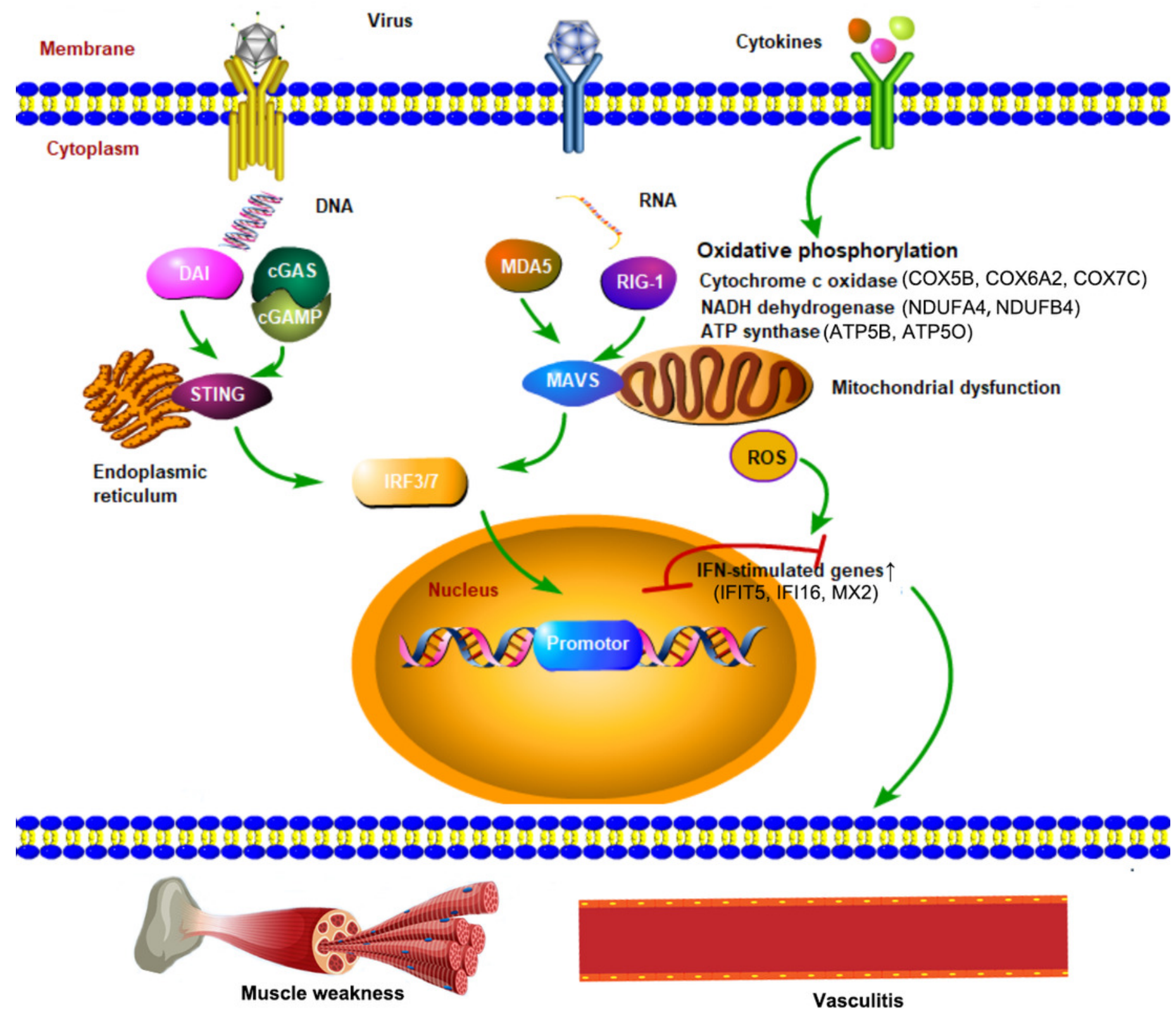
Table $\mathbf{1}$ (on next page)

Table 1 
Table 1. Hub genes identified by co-expression and MCODE methods

2

\begin{tabular}{|c|c|c|c|c|c|}
\hline Gene & GS.JDM & MM & MCODE_Score & regulation & module \\
\hline ATP5O & -0.860 & 0.905 & 7.0 & down & blue \\
\hline АТР5B & -0.903 & 0.892 & 7.0 & down & blue \\
\hline NDUFV2 & -0.792 & 0.912 & 7.0 & no sig & blue \\
\hline NDUFB10 & -0.844 & 0.946 & 7.0 & NA & blue \\
\hline $\mathrm{COX} 7 \mathrm{C}$ & -0.784 & 0.919 & 7.0 & down & blue \\
\hline COX5B & -0.830 & 0.937 & 7.0 & down & blue \\
\hline NDUFB4 & -0.805 & 0.922 & 6.0 & down & blue \\
\hline COX6A2 & -0.841 & 0.922 & 7.0 & down & blue \\
\hline NDUFA4 & -0.833 & 0.833 & 7.0 & down & blue \\
\hline VDAC1 & -0.880 & 0.896 & 4.8 & down & blue \\
\hline SLC25A3 & -0.859 & 0.903 & 6.0 & down & blue \\
\hline MDH2 & -0.851 & 0.921 & 5.0 & down & blue \\
\hline TPI1 & -0.899 & -0.899 & 2.7 & down & blue \\
\hline RPL21 & -0.827 & 0.915 & 0.5 & down & blue \\
\hline MRPS7 & -0.842 & 0.916 & 2.0 & no sig & blue \\
\hline PARP9 & 0.844 & 0.950 & 1.2 & NA & lightgreen \\
\hline DTX3L & 0.844 & 0.961 & 2.0 & NA & lightgreen \\
\hline MX2 & 0.930 & 0.913 & 3.0 & up & lightgreen \\
\hline SAMHD1 & 0.846 & 0.954 & 2.0 & up & lightgreen \\
\hline RNF213 & 0.841 & 0.961 & 2.0 & NA & lightgreen \\
\hline EIF4B & -0.885 & -0.919 & 0.0 & down & lightgreen \\
\hline IFIT5 & 0.934 & 0.921 & 3.0 & up & lightgreen \\
\hline SP110 & 0.864 & 0.963 & 0.7 & up & lightgreen \\
\hline IFI16 & 0.881 & 0.913 & 3.0 & up & lightgreen \\
\hline PLSCR1 & 0.879 & 0.922 & 3.0 & up & lightgreen \\
\hline EPSTI1 & 0.797 & 0.925 & 2.0 & NA & lightgreen \\
\hline DDX60L & 0.846592 & 0.923402 & 0.0 & NA & lightgreen \\
\hline CLIC1 & 0.768 & 0.933 & 2.0 & up & midnightgreen \\
\hline
\end{tabular}

3

4 
Table 2 (on next page)

Table 2 
Table 2. Small molecule drugs screening based on CMap database

2

\begin{tabular}{lrrrrrr}
\hline Cmap name and cell line & $\begin{array}{l}\text { Mean } \\
\text { score }\end{array}$ & Number & Enrichment & $\boldsymbol{P}$-value & Specificity & Percent non-null \\
\hline 0175029-0000 & -0.696 & 6 & -0.982 & 0 & 0 & 100 \\
acacetin & -0.465 & 6 & -0.785 & 0.00018 & 0 & 100 \\
helveticoside & -0.494 & 6 & -0.754 & 0.00044 & 0.013 & 83 \\
lanatoside C & -0.424 & 6 & -0.7 & 0.00167 & 0.0638 & 66 \\
betonicine & 0.239 & 6 & 0.676 & 0.00312 & 0.0065 & 66 \\
deferoxamine & -0.345 & 8 & -0.556 & 0.00726 & 0.0184 & 62 \\
famprofazone & -0.203 & 6 & -0.557 & 0.02862 & 0.1035 & 50 \\
felodipine & 0.213 & 7 & 0.511 & 0.03088 & 0.1089 & 71 \\
trichostatin A - HL60 & 0.283 & 34 & 0.623 & 0 & 0.0798 & 52 \\
trichostatin A - MCF7 & 0.172 & 92 & 0.399 & 0 & 0.673 & 56 \\
LY-294002 - PC3 & -0.243 & 12 & -0.494 & 0.00325 & 0.2249 & 58 \\
valproic acid - HL60 & 0.254 & 14 & 0.417 & 0.01034 & 0.1812 & 50 \\
sirolimus - HL60 & 0.305 & 10 & 0.534 & 0.00359 & 0.0347 & 70 \\
vorinostat - MCF7 & 0.22 & 7 & 0.5 & 0.0378 & 0.7655 & \\
\hline \multicolumn{1}{c}{3} & & & & & 71 \\
\hline
\end{tabular}

3 